\title{
EARLY DETECTION, APPROPRIATE MANAGEMENT - ENSURE A LEGACY OF GOOD HEALTH
}

\author{
Amir SK \\ Penang Medical College \& Consultant Endocrinologist, Penang Hospital
}

The current volume of JUMMEC contains a wide spectrum of health topics highlighting the importance of controlling metabolic factors in diabetes, selection of heart failure patients for innovative treatment, early postoperative analgesia, testing of a translated screener for urinary tract problems, problems when commissioning a new operating theatre and breastfeeding issues.

Chronic diseases such as heart disease, and diabetes account for a large proportion of health care expenditure and these can be prevented (or at least delayed) by healthier diets, improved physical fitness and, in the case of the former, no tobacco use. Screening tests exist for many chronic diseases which can detect the disease while they are still asymptomatic. Early diagnosis and appropriate treatment of disease may lead to cures and better survival. Type 2 diabetes mellitus (T2DM) is an ideal disease for early detection with simple measurement of timed blood glucose. Epidemiological studies on T2DM and the related metabolic syndrome have consistently detected undiagnosed individuals for every known individual with diabetes. There are known risk factors that increase the likelihood of developing T2DM with family history, excess body weight, and previous gestational diabetes mellitus being prominent. T2DM is an important health care problem as it can lead to long term microvascular as well as cardiovascular complications.

The review article by Vijay and Chan examines the link between glycaemia and complications (1). For those treating individuals with diabetes, their intuition would make them pursue normalisation of the blood sugar. It was formally confirmed in the 1990s that in type and 1 and 2 DM, DCCT and UKPDS respectively, reducing glycaemia leads to improvements in microvascular outcome. The picture for cardiovascular disease benefit was less clear, with several unanswered questions like level of glycated haemoglobin (HbA1c) lowering needed, safety of rapid lowering of $\mathrm{HbA} 1 \mathrm{c}$ in all individuals and whether all glucose lowering agents are safe and efficacious. Coronary heart disease is the major cause of morbidity and mortality in patients with T2DM. Vijay and Chan mentioned three trials with largely negative CV benefits, namely ACCORD, ADVANCE and VADT, where the studies have not demonstrated a benefit from intensive glycaemic control on primary CVD outcomes. Hypoglycaemia was worse in the intensive arm of ACCORD, a study stopped because of excess mortality in the intensive group. A recent metaanalysis looking at UKPDS, ADVANCE, ACCORD, VADT as well as PRO-active (Kausik Ray et al, Lancet 2009; 373: 1765-72) would suggest that it is not correct to conclude that glucose control has no part to play to address CHD risk. They showed a significant $17 \%$ and $15 \%$ reductions in events of non-fatal myocardial infarction and coronary heart disease respectively with better control of blood sugar (mean $\mathrm{HbA} 1 \mathrm{c} 0.9 \%$ lower in intensive arm). There was no impact on stroke or all-cause mortality. Of interest, ACCORD and PROactive as subgroups showed increase in heart failure (due to fluid retention) but this is not seen in all groups reflecting the glucose-lowering drugs used in these two trials. Overall, there is also a trend of higher non-CHD CV death in the intensive group, which could reflect arrhythmias. However, the UKPDS 10 year follow-up of patients who were given intensive glucose control (achieving $\mathrm{HbA} 1 \mathrm{c} \sim 7.0 \%$ ), suggest that initial intensive therapy followed by reasonable glycaemic control lead to clear macrovascular benefits not seen in the initial study period. They showed that despite early loss of glycaemic differences (after two years) between intensive and conventional groups, a continued reduction in microvascular risk and emergent risk reductions for myocardial infarctions and deaths from any cause were observed during 10 years of post-trial follow-up. This would suggest that benefit from intensive glucose control on CVD takes a longer duration to manifest. To get CVD benefit hyperglycaemia must be controlled early after diagnosis and continued over a long term. As highlighted by the authors, an $\mathrm{HbA} 1 \mathrm{c}$ goal of $7.0 \%$ for most patients seems reasonable and safe. This goal can be adjusted

\footnotetext{
Correspondence :

Amir S Khir

Dean and Foundation Professor of Medicine

Penang Medical College \& Consultant Endocrinologist

Penang Hospital

Email:amir@pmc.edu.my
} 
to $<6.5 \%$ for younger patients with no $\mathrm{CHD}$ and no risk associated with hypoglycaemia, and less tight control in those in whom CVD risk was higher. As mentioned by the authors, therapies directed at multiple related conditions such as dyslipidaemia, hypertensions, hypercoagulable state, obesity, IR have to be undertaken. The Steno-2 showed that in type $2 \mathrm{DM}$, intensive intervention with multiple drug combinations and behaviour modifications had sustained effect with respect to vascular complications and rates of death including that due to CVD

Zul Hilmi pointed out that the criteria for selecting heart failure patients in whom cardiac resynchronisation therapy (CRT) should be looked at again (2). Using broad QRS duration alone to indicate presence of ventricular dyssynchrony can miss detecting patients suitable for CRT depriving them of this useful therapy. He suggests that measurements (SD of time to peak systolic velocity in 12 segments, Ts-SD-12) using the Tissue Doppler Imaging be used, being a superior criterion to predict response to CRT. He found that in patients with significant dyssynchrony, there was no significant correlation between QRS duration and the Ts-SD-12.

The treatment of hyperthyroidism (Graves' disease mainly) has changed little over 60 years with thioureylene drugs-carbimazole, methimazole and propylthiouracil, radioactive iodine therapy and surgery being the mainstay. The thioureylenes reduce thyroid hormones and favour immunological remission via direct or indirect effects. However, long term remission only occurs in $40-50 \%$ of cases, and many patients will need the other mentioned modalities. Vijay has described a case of aplastic anaemia more commonly associated with perchlorate (3). On the whole, agranulocytosis is rare $(0.1-0.4 \%)$ and aplastic anaemia is very rare. It is important to note the potential for cross reactivity between CMZ and PTU. In Graves' disease the use of immunomodulation seems a logical therapy. Systemic steroids are not used except in severe eye disease, but there is a recent study from China looking at impact of intrathyroid dexamethasone on remission rates (XM Mao et al, J Clin Endocrinol Metab 2009; 94: 4984-4991). The use of rituximab has not caught on with few studies showing benefit.

Nazatul has demonstrated challenges encountered by breastfeeding mothers in a preliminary qualitative research (4). She has identified several obstacles. Measures must be taken to improve knowledge on breastfeeding, increase support from health professionals, parents and siblings and improve facilities to express milk and to breastfeed at the work place and public places. More hospitals should become Baby Friendly in the real sense. It would be useful for these measures to be put in motion and her study repeated to see whether the implementation is successful or not.

Marzida Mansor identified the need of providing effective postoperative analgesia to mothers following Caesarean Section as the freedom from pain must be achieved without sacrificing mobility and mental alertness (5). She studied regular oral NSAID drug, diclofenac against the usual parenteral pethidine, an opioid type drug, in a randomised manner. Once the effect of spinal anaesthesia is over, patients took twice daily diclofenac orally versus subcutaneous pethidine. It would appear that there is still a need for the subcutaneous pethidine on the first postoperative day but the NSAID drug is adequate on the second and third day. As expected the NSAID does not cause sedation and received high overall satisfaction scores. A policy for giving opioids should be relooked so that the use can be rational rather than continued as a routine when the less centrally suppressive therapy can be just as useful.

Mafeitzeral wrote about experience in commissioning a new operating theatre at The University of Malaya Medical Centre (UMMC) Trauma Centre (6). Problems encountered were not all anticipated, but could be solved. International standards needed to be followed though they could not be adhered to entirely. The experiences recounted should be useful for others who are commissioning an operating theatre.

Muhilan described the construction and validation of a translated Overactive Bladder Screener for assessing urinary tract (7). $O A B$ v8 was translated into Bahasa Malaysia but like all translated document, it is important that the translated version remains valid, accurate and retains the sensitivity and specificity of the screening test. The translated version was done by conventional means with back translation and review by a panel of experts. When tested in two groups of patients in a pilot study, the questionnaire demonstrated good internal consistency for component items and overall score. It was able to detect diagnostic items in both symptomatic and asymptomatic patients. It is considered to be suitable for use. Continuous scrutiny of this new tool should be practised and results of a larger sample should be analysed. Further modification of the translated version may be required. 


\section{References}

1. Vijay AP, Chan SP. Diabetes control-the legacy of a memory. JUMMEC 2009; 12(2): 47-56.

2. Yaacob ZH, Syed Tamin S, Nik Zainal NH, Chee $\mathrm{KH}$, Chong WP, Hashim NE, et al. Ventricular Dyssynchrony is common among heart failure patients with narrow QRS complex. JUMMEC 2009; 12(2): 57-62.

3. Vijay AP, Lim SS, Tan ATB, Rokiah P, Chan SP. Carbimazole-induced aolastic anaemia-a case report. JUMMEC 2009; 12(2): 63-66.

4. NazatulSB.Challenge and supportfor breastfeeding in highly motivated Malaysian mothers. JUMMEC 2009; 12(2): 63-66.
5. Marzida M. A randomized controlled study comparing subcutaneous pethidine with oral diclofenac for pain relief after caesarean section. JUMMEC 2009; 12(2): 67-73.

6. Mafeitzeral M, Chan L. Ensuring patient safety in the new operating theatre of UMMC Trauma Centre. An anaesthesiologist novice experience. JUMMEC 2009; 12(2): 74-82.

7. Muhilan AP, Norman D, Sanjay R, Azah R. Construction and validation of a Malay version of the Overactive Active Screener for assessing urinary tract symptoms in a Malaysian population. JUMMEC 2009; 12(2): 83-91. 